\title{
Mobile Robot's Map Reconstruction Based on DSmT and Fast-Hough Self-Localization
}

\author{
Xinhan Huang, Xinde Li, Zuyu Wu, Min Wang \\ Department of Control Science and Engineering, Huazhong University of Science and Technology \\ Wuhan 430074 , China \\ xhhuang@mail.hust.edu.cn
}

\begin{abstract}
This paper presents a new tool based on DSm T (Dezert-Smarandache Theory) and fast-Hough self-localization which is applied to autonomous mobile robot's map reconstruction. Uncertainty from sonar information is fused and managed in rebuilding grid map effectively with DSmT, since fast-Hough self-localization from laser information overcomes some shortcomings of classical Hough transformation including high amount of computation and low precision of localization. When a virtual mobile robot evolves in the virtual environment with different outline features, the result can adequately testify that the new tool owns better performance to rebuild map. In general, this research provides an approach for studying autonomous navigation of mobile robot or unmanned land vehicle (ULV) in unknown environment, also provides a humancomputer interactive interface to manage and manipulate the robot remotely.
\end{abstract}

Index Terms - Map Reconstruction, DSmT, Self-localization, Hough transformation.

\section{INTRODUCTION}

The exploration in entirely unknown environment for intelligent mobile robots has been a popular and important subject. Map reconstruction is a very important part in this subject. Presently, mainly there are three mapping methods: grid map, geometrical feature map and topological map. Grid map is the most simple and popular method among them. There are many reports about it, for example, Elfes, and Moravec (1985) [1] firstly represented the probability of the grid occupied by obstacles with probability theory, which was called as occupancy grid method later [2]. It is very popular since its simpleness in realization and low amount of computation, while it has low precision in map reconstruction. Giuseppe Oriolo et al. (1997) [3]firstly proposed a method of map reconstruction based on fuzzy logic, which has high precision in loose environment, while it spends more time in the same environment than that with occupancy grid method. Daniel Pagac (1996) [4] firstly applied DST [5] to map reconstruction for mobile robots, where Dempster's combinational rule is used to redistribute the belief mass of grid occupancy reasonably. However, it has a fatal shortcoming that it can't deal with the highly conflictive information. Weihua Wang et al. (2003) [6] proposed a new grid method based on grey system theory, which has a high precision and moderate amount of computation, while its detecting range is very small. We proposed to apply DSmT[7] to grid map reconstruction in 2006[8][9], though it has overcome the shortcoming of DST[10][11], its precision still need to be improved further, since it didn't consider redistributing the conflicting mass with PCR rule [12] or other rules, and even didn't yet consider the self localization. In this paper we give a further research on map reconstruction based on DSmT (i.e. DSmC and PCR5 rules) and Fast-Hough Self-Localization in a static environment.

This paper is organized as follows: In section II, Simple review of DSmT. In section III, A model based on DSmT with sonar information is given. In section IV, A new self -localization based on Fast-Hough transformation is proposed. In section V, A simulation experiment is done to testify the performance of new tool in map reconstruction under self localization. At last, a conclusion is reached in section VI.

\section{REVIEW OF DSMT}

DSmT (Dezert-Smarandache Theory) [7]is a new, general and flexible theory of information fusion, which can solve the fusion problems of different tiers including data-tier, featuretier and decision-tier. It not only can dispose the static problem of fusion, but also can dispose the dynamic one. It has a prominent merit that it can deal with the highly conflicting information according to Proportional Conflict Redistribution rule no. 5 (PCR5)*, which goes backwards on the tracks of the conjunctive rule and only redistributes proportionally the partial conflicting masses to the sets involved in the conflict by considering the conjunctive normal form of the partial conflict[12].

\section{A. Basic of DSmT}

1) Let $\Theta=\left\{\theta_{1}, \theta_{2}, \cdots, \theta_{n}\right\}$, here $\Theta$ is a frame of discernment, which includes $\mathrm{n}$ finite focal elements $\theta_{i}(i=1, \cdots n)$. Because the focal element is not precisely defined and separated, so that no refinement of $\Theta$ in a new larger set $\Theta_{\text {ref }}$ of disjoint elementary hypotheses is possible.

2) The hyper-power set $D^{\Theta}$ is defined as the set of all compositions built from elements of $\Theta$ with $U$ and $\cap$ ( $\Theta$ generates $D^{\Theta}$ under operators $\cup$ and $\cap$ ) operators such that

$$
\text { a) } \phi, \theta_{1}, \theta_{2}, \theta_{3} \cdots \theta_{n} \in D^{\Theta}
$$

\footnotetext{
* Dezert and Smarandache have proposed 5 versions of proportional conflict redistribution rules, which become one part of DSmT.
} 
b) If $A, B \in D^{\Theta}$, then $A \cap B \in D^{\Theta}$ and $A \cup B \in D^{\Theta}$

c) No other elements belong to $D^{\Theta}$, except those obtained by using rules a) or b).

3) General belief function and Plausibility function Let $\Theta=\left\{\theta_{1}, \theta_{2}, \cdots, \theta_{n}\right\}$ is the general frame of discernment. For every evidential source $\mathrm{S}$, let us define a set of map of $m(\cdot): D^{\Theta} \in[0,1]$ associated to it (abandoning Shafer's model) by assuming here that the fuzzy/ vague/ relative nature of elements $\theta_{i}(i=1,2,3 \cdots n)$ can be non-exclusive, as well as no refinement of $\Theta$ into a new finer exclusive frame of discernment $\Theta_{\text {ref }}$ is possible. The mapping $m($.$) is called a$ generalized basic belief assignment function (gbbaf), if it satisfies

$$
m(\phi)=0 \quad \text { and } \quad \sum_{A \in D^{\Phi}} m(A)=1
$$

The general belief function and plausibility function are defined respectively in almost the same manner as within the DST, i.e.

$$
\begin{gathered}
\operatorname{bel}(A)=\sum_{B \in D^{\ominus}, B \subseteq A} m(B) \\
\operatorname{Pl}(A)=\sum_{B \cap A \neq \phi, B \in D^{\Theta}} m(B)
\end{gathered}
$$

4) Classical (free) DSmT rule of combination

Let $M^{f}(\Theta)$ is a free model of DSmT, and then the classical (free) DSmT rule of combination for $k \geq 2$ sources is given as follows:

$$
\forall A \neq \phi \in D^{\Theta}, \quad=\sum_{\substack{X_{1}, \cdots X_{k} \in D^{\Theta} \\\left(X_{1} \cap \cdots X_{K}\right)=A}} \prod_{i=1}^{m_{M^{f}(\Theta)}(A) \cong\left[m_{1} \oplus \cdots m_{k}\right](A)} m_{i}\left(X_{i}\right)
$$

\section{B. Proportional Conflict Redistribution Rule}

PCR5 fines the conflicting mass, and just redistribute the partial conflicting mass to the elements involved in the partial conflict, in order to improve the quality of fusion [12].

Here we introduce the PCR5 formula for $s=2$ sources: $\forall X \in G /\{\phi\}$,

$$
\begin{aligned}
& m_{P C R S}(X)=m_{12}(X)+ \\
& \sum_{Y \in G /\{X\}}\left[\frac{m_{1}(X)^{2} m_{2}(Y)}{m_{1}(X)+m_{2}(Y)}+\frac{m_{2}(X)^{2} m_{1}(Y)}{m_{2}(X)+m_{1}(Y)}\right]
\end{aligned}
$$

where, $c(X)$ represents the canonical form of $X, m_{12}(\bullet)$ corresponds to the conjunctive consensus, i.e. $m_{12}(X) \cong \sum_{\substack{X_{1}, X_{2} \in G \\ X_{1} \cap X_{2}}} m_{1}\left(X_{1}\right) m_{2}\left(X_{2}\right)$, and where all denominators are different from zero. If a denominator is zero, that fraction is discarded. Please see also [10], if $s>2$.

\section{- :ModELING For SonAR GRID INFORMATION}

Sonar sensors' working principle (shown in Fig. 1) is: producing sheaves of cone-shaped wave, and detecting the objects by receiving the reflected wave. Due to the restriction of sonar physical characteristic, metrical data behaves out uncertainty as follows:

a) Beside its own error of making, the influence of external environment is also very great, for example, temperature, humidity, atmospheric pressure and so on.

b) Because the sound wave spreads outwards in the form of loudspeaker, and there exists a cone-shaped angle, we cannot know the true position of object detected among the fan-shaped area, with the enlargement of distance between sonar and it.

c) The use of lots of sonar sensors will result in interference each other. For example, when the $i$ th sonar gives out detecting wave towards an object of irregular shape, if the angle of incidence is too large, the sonar wave might be reflected out of the receiving range of the $i$ th sonar sensor or also might be received by other sonar sensors.

d) Because sonar sensors utilize the reflection principle of sound wave, if object absorbs very heavy sound wave, the sonar sensor might be invalid.

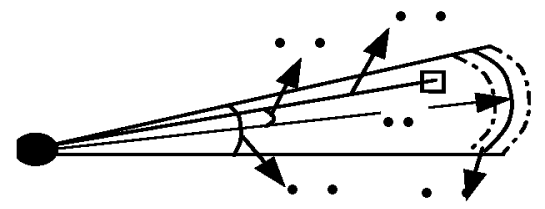

Fig. 1 The sketch of the principle of sonar

\section{A. Modeling Based on DSmT}

Pointing to the characteristics of sonar's measurement, we construct a model of uncertain information acquired from grid map using sonar based on DSmT. Here we suppose there are two focal elements in system, that is $\Theta=\left\{\theta_{1}, \theta_{2}\right\}$, here $\theta_{1}$ means grid is empty, $\theta_{2}$ means occupied, and then we can get its hyper-power set $D^{\Theta}=\left\{\phi, \theta_{1} \cap \theta_{2}, \theta_{1}, \theta_{2}, \theta_{1} \cup \theta_{2}\right\}$. Every grid in environment is scanned $k \geq 2$ times, each of which is viewed as source of evidence. Then we may define a set of map aiming to every source of evidence and construct the general basic belief assignment functions (gbbaf) as follows: $m\left(\theta_{1}\right)$ is defined as the gbbaf for grid-unoccupied (empty); $m\left(\theta_{2}\right)$ is defined as the gbbaf for grid-occupied; $m\left(\theta_{1} \cap \theta_{2}\right)$ is defined as the gbbaf for holding grid-unoccupied and occupied simultaneous (conflict). $m\left(\theta_{1} \cup \theta_{2}\right)$ is defined as the gbbaf for grid-ignorance due to the restriction of knowledge and experience presently (here referring to the gbbaf for these grids still not scanned presently ), it reflects the degree of ignorance of grid-unoccupied or occupied.

The gbbaf of a set of map $m():. D^{\oplus} \rightarrow[0,1]$ is constructed by authors such as the formulae (5) (8) according to sonar physical characteristics.

$$
\begin{aligned}
& m\left(\theta_{1}\right)=E(\rho) \cdot E(\theta)= \\
& \begin{cases}\left(1-(\rho / R-2 \varepsilon)^{2}\right) \cdot(1-\lambda / 2) & \text { if }\left\{\begin{array}{l}
R_{\min } \leq \rho \leq R-2 \varepsilon \\
0 \leq \theta \leq \omega / 2
\end{array}\right. \\
0 & \text { otherwise }\end{cases}
\end{aligned}
$$




$$
\begin{aligned}
& m\left(\theta_{2}\right)=\mathrm{O}(\rho) \mathrm{O}(\theta)= \\
& \left\{\begin{array}{cl}
\exp \left(-3 \rho_{\mathrm{v}}(\rho-\mathrm{R})^{2}\right) \cdot \lambda \text { if } & \left\{\begin{array}{l}
R_{\min } \leq \rho \leq R+\varepsilon \leq R_{\max } \\
0 \leq \theta \leq \omega / 2
\end{array}\right. \\
0 & \text { otherwise }
\end{array}\right. \\
& m\left(\theta_{1} \cap \theta_{2}\right)= \\
& \left\{\begin{array}{cc}
\left(1-(2(\rho-(R-2 \varepsilon)) / R)^{2}\right) & \text { if }\left\{\begin{array}{l}
R_{\min } \leq \rho \leq R \leq R_{\max } \\
0 \leq \theta \leq \omega / 2
\end{array}\right. \\
0 & \text { otherwise }
\end{array}\right. \\
& m\left(\theta_{1} \cup \theta_{2}\right)= \\
& \begin{cases}\tanh (2(\rho-R)) \cdot(1-\lambda) & \text { if }\left\{\begin{array}{l}
R \leq \rho \leq R+2 \varepsilon \\
0 \leq \theta \leq \omega / 2
\end{array}\right. \\
0 & \text { otherwise }\end{cases} \\
& \lambda=E(\theta)=Q(\theta)=\left\{\begin{array}{ccc}
1-(2 \theta \mid \omega)^{2} & \text { if } & 0 \leq|\theta| \leq \omega \mid 2 \\
0 & \text { otherwise }
\end{array}\right.
\end{aligned}
$$

where $E(\theta)=1-\lambda / 2, O(\theta)=\lambda, E(\rho)=\left(1-(\rho / R-2 \varepsilon)^{2}\right)$, $\mathrm{O}(\rho)=\exp \left(-3 \rho_{\mathrm{v}}(\rho-\mathrm{R})^{2}\right)$, where $\lambda$ is given by (9) (also see ref. [1] for justification).

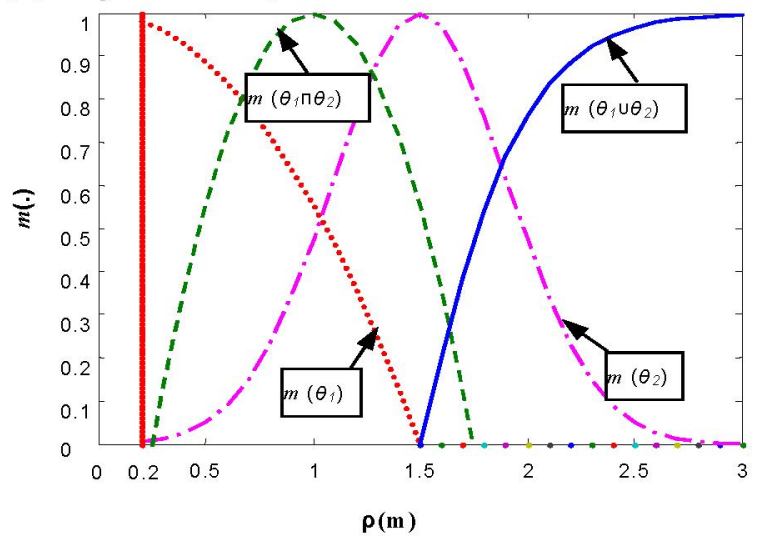

Fig. $2 m($.$) as function of \rho$ given by formulae (5 -8)

From Fig. 2 we can see that gbbaf reflects really the characteristics of sonar information with the shift of $\rho$ in the course of grid map reconstruction. Here we assume the detecting range of sonar sensor from $0.2 \mathrm{~m} \sim 3 \mathrm{~m}$. High conflictive information happens about at the point of intersection of two curves between $m\left(\theta_{1}\right)$ and $m\left(\theta_{2}\right)$. The maximum $m\left(\theta_{2}\right)$ happens at $\rho=1.5 \mathrm{~m}$, while the maximum of $m\left(\theta_{1}\right)$ happens at $\mathbf{R}_{\min }=0.2 \mathrm{~m}$. In order to satisfy the definition of DSmT, and assure the sum of all mass of $m(\cdot)$ to be one, we must renormalize them while acquiring sonar grip information.

\section{FAST-HOUGH SELF-LOCALIZATION}

\section{A. The Principle of Fast-Hough Transformation}

It is well known that classical Hough transformation plays an important role in picking up straight line feature since it was proposed by Duda and Hart in 1972 [13]. However, it doesn't satisfy the real-time requirement in self - localization due to the high amount of computation. We here put forward an improved Fast-Hough transformation.
The basic principle is when mobile robot moves to a certain position, we cluster all scanned points in the detecting range of laser range finder ( $0 \sim 180$ degrees), and classify them into subset $S_{i}(i \in 1,2 \cdots)$. Supposed that there are $n$ points in the subset $S_{i}$, then compute respectively the slope between every two points. Setting up a counter, if the slope is in the setting range, we add one on the counter. When finished the statistic of all points in the subset $S_{i}$, we classify all points having the maximum number to support a certain slope into a new subset $S_{i}^{a}$. We can pick up the straight line $l_{i j, j \in(1,2 \cdots)}$ through Hough transformation and then find those points owning the sub maximum number and put them into another subset $S_{i}^{b}$. Also pick up the second straight line until the number to support a certain slope is lower than the threshold. The mentioned above is the key difference from other Fast transformation methods [14]. Then we begin to deal with another subset $S_{j}$ by same way until we finish all points scanned in the detecting range of laser. By doing this we not only reduce the complexity and amount of computation, but also improve the precision of picking up straight line, as well as find smaller features which might be omitted by other Hough transformation methods.

\section{B. Self-localization of Robot's Pose}

In a static and structured environment, if we don't consider the small accumulative error and metrical error, then the coordinate of whole object outline in the environment detected by laser range finder on mobile robot will be consistent completely with the real one in global coordinate system. In fact, it is impossible because of inaccurate robot's pose. So robot's pose must be calibrated. Of course, if mobile robot runs in the dynamic or non-structured environment, it is very difficult to pick up some straight line features only using Hough transformation method. In this case we must consider mixing with other effective ways [15].

In our research the main idea about self-localization of mobile robot with Fast-Hough transformation method is Step- by Step mode. That is, when mobile robot moves a step, its pose can be calibrated with the referenced straight line and gets a new pose. According to the new pose calibrate some new straight line segments detected and choose some of them as the new referenced straight lines, and so on.

In order to compare lines in the course of self calibration we define some attributes about a line, i.e. Line $\left(\rho_{l}, \theta, n, l, s p\right.$, $e p, d i r, d)$ shown in Fig.3. Where $\rho_{l}$ is the distance $O P$ between the origin $O$ and the feature straight line $A B . \theta$ is the angle of inline between the perpendicular $O P$ and the positive direction of axis $\mathrm{X}(0 \sim 360$ degrees $)$. $n$ is the point number including all points which may be fitted into a feature straight line. $s p$ is the starting point of feature straight line $A B$. ep is the endpoint. $d i r$ is the observation direction relying on the location between robot and feature straight line $A B$ i.e. $\operatorname{dir}=\operatorname{sign}\left(\rho_{l}-x \cos (\theta)-y \sin (\theta)\right)$, where $\operatorname{sign}$ is 
the Sign function. If robot is nearer the origin than the feature straight line, then $d i r=1$; if robot is farther away from the origin than the feature straight line $A B$, then $d i r=-1$; if robot lied on the feature straight line, then $\operatorname{dir}=0 . d$ is the distance between robot and feature straight line.

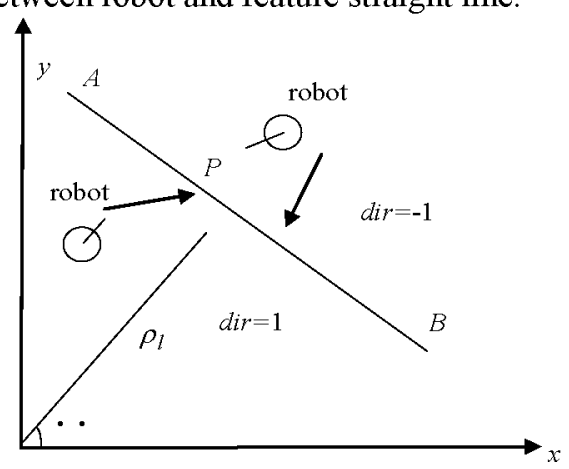

Fig.3 The observation direction for feature line

In order to justify whether a feature straight line is similar to another one we must propose the following four necessary conditions.

(a) Justify whether the observation direction is consistent.

(b) Whether the intersection angle of two feature straight lines is smaller than a setting threshold.

(c) Whether the distance from the endpoint of one line segment to another feature straight line is smaller than a setting threshold.

(d) Justify whether two lines are independent by projecting line segment 1 to line segment 2. If the projective line segment has a common part with line segment 2 the two line segments might be not independent.

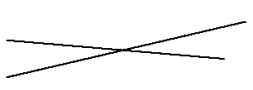

A. Both lines are similar

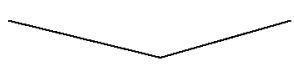

C. Both lines aren't similar

Fig. 4 Some examples about the similarity between two straight lines

We give several examples to justify whether a feature straight line is similar to another one according to the aforementioned conditions. For example, two feature straight lines are similar in Fig.4.A. The two feature straight lines aren't similar in Fig.4.B, C and D because B satisfies condition (d) and doesn't satisfy condition (c), C doesn't satisfy condition(b)and (d), D doesn't satisfy condition (b) and (c).

In general, there are two main processes in the course of self localization, one is the mergence of line segments and the other is calibration of robot's pose which isn't independent each other.

1) The mergence of line segments
When mobile robot moves along a very long wall which is out of the range of laser, thus the laser range finder can't detect the whole wall in one time. In this case, the mergence between new line segment and the old one is very necessary. We must justify whether the two line segments are on the common line according to the similar conditions. The simple way to merge two line segments is shown in Fig.5. To extend the referenced straight line we first project the new detecting straight line $\mathrm{AB}$ to the relative referenced line $\mathrm{EF}$ and then the two endpoints of projection line segment $\mathrm{CD}$ can be reached. After a simple sort of four endpoints (C, E, D, F) justify whether the distance $\mathrm{CM}$ is bigger than $\mathrm{EM}$, here $\mathrm{M}$ is the midpoint of referenced line segment EF. If yes, then we will extend $\mathrm{EF}$ to point $\mathrm{C}$, and get the new line segment $\mathrm{CF}$. In this course we must consider the process of self calibration because correct pose is the key of merging two line segments.

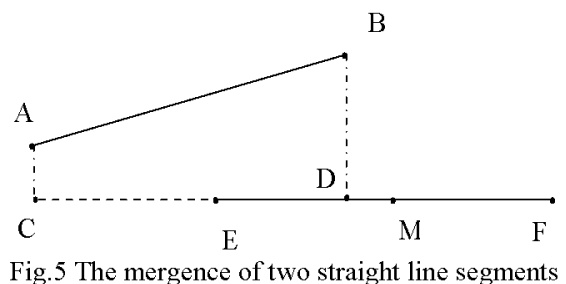

2) Self calibration

Suppose the initial localization of mobile robot is correct, then the detecting feature straight lines are taken as the referenced straight line (generally speaking we choose one or several longer straight lines) and add them into the historical list of referenced straight line which is the real outline of the object in the environment theoretically. When robot moves a bit, get some old feature straight lines near referenced lines which might coincide completely or partially with these referenced straight lines. Then compute the deviation angle and distance between the new detecting line segment and the corresponding referenced one and calibrate the robot's pose. At the same time we also get some new feature straight lines and calibrate them according to new pose of robot. At last choose new longer straight lines and add them into historical list of referenced lines, and so on. Thus the robot is able to finish the task of self-localization in a perfect environment.

\section{Simulation Experiments}

The experiments consist of simulating the autonomous navigation for Pioneer II Robot with 16 sonar detectors and a laser range finder. The map reconstruction with sonar sensors on the mobile robot is built in a static environment shown in Fig.6 using SRIsim simulator supplied by ActivMedia Company. In general there are some different outlines such as circle, rectangle, and triangle. The experiments based on the improved Fast Hough transformation are done in our developed platform for intelligent perception fusion system. The platform developed using Visual $\mathrm{C}++6.0$ and OpenGL is a client end shown in fig.7 which can connect the server end. When the virtual robot runs in the virtual environment the server end can collect a lot of information from the SRIsim 
including the location of robot, sensors reading, velocity etc. By the protocol of TCP/IP the client end can get any information from the server end. The Pioneer II Robot may begin to run at arbitrary location. Suppose the beginning location is of $300 \mathrm{~mm}$ in $\mathrm{X}$ direction and $2300 \mathrm{~mm}$ in $\mathrm{Y}$ direction with 0 degree angle the robot faces to. Using grid method and our improved method we get the original environment and reconstructed map as shown in Fig. 6 and Fig. 7 respectively.

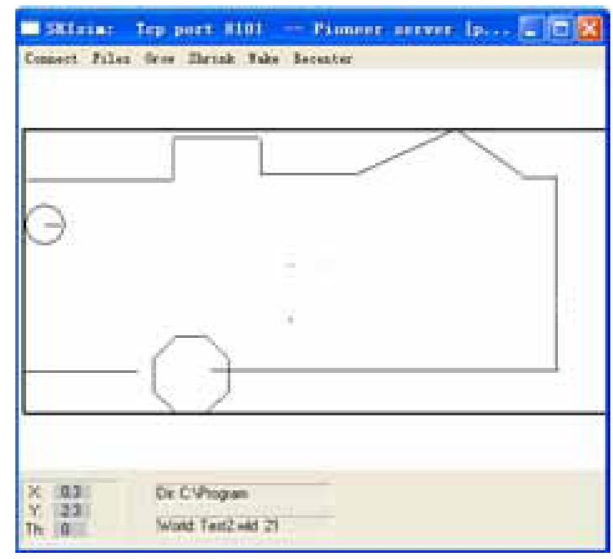

Fig.6 The static environment in SRIsim simulator

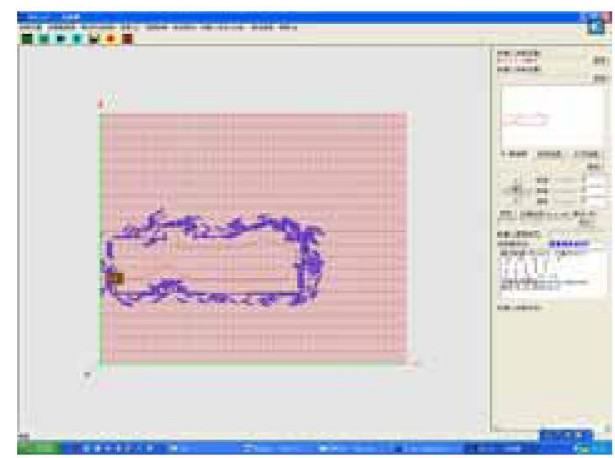

Fig. 7 The platform for intelligent perception fusion system

To describe the experiment clearly, the main steps of procedure are given as follows.

1) Initialize the parameters of robot including initial location, moving velocity, etc.

2) When the robot is running, acquire 16 sonar readings, laser reading and get new robot's location and calibrate it with Fast-Hough transformation described in detail in section IV. (Here we set the first time stamp, its measurement period used in our experiments is $100 \mathrm{~ms}$ )

3) Compute gbbaf of the fan-form area detected by each sonar sensor according to formula (5) to formula (8).

4) Whether some cells are scanned renewedly by sonar sensors (same sonar in deferent location, or different sonar sensors, of course, here we suppose each sonar sensor has the same characteristics)? If yes go to next step, otherwise go to step 6 .

5) According to the combination rule and the PCR5 in formulae (3) and (4) respectively we can get the new basic belief masses and redistribute the conflict mass to the new basic belief masses.

6) Compute the credibility of occupancy $\operatorname{bel}\left(\theta_{2}\right)$ of some grids which have been fused according to formula (1).

7) Update the map of the environment. (Here we set the second time stamp, its measurement period used in our experiments is also $100 \mathrm{~ms}$ ) Whether the map reconstruction has been finished? If yes stop the mobile robot and exist. Otherwise go to step 2 .

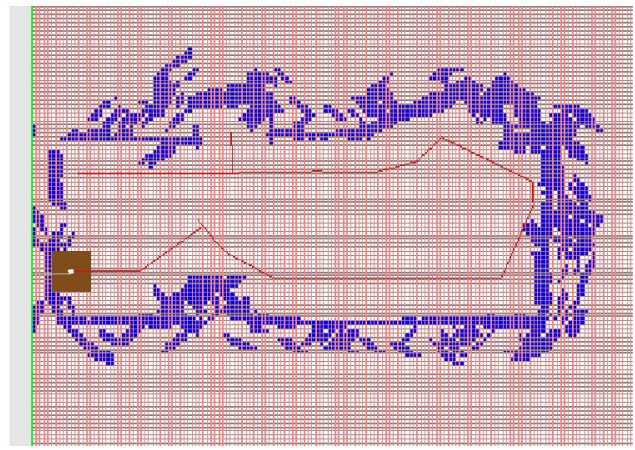

Fig. 8 Map reconstruction with DSmT under self localization

Finally we reconstruct the map shown in the Fig.8 with the new tool. It can be concluded that:

1) From the Fig. 8 we can see that the new tool has a better performance in building map (i.e. more distinct outline including circle, rectangle, and triangle) than just using the method of DSmT[8][9], because of considering the conflict factor and redistributing the conflict masses to other basic belief masses according to the PCR5.

2) The restrained spreading arithmetic overcomes the shortcoming of the global grids which must be reckoned once for sonar scanning every time and improves the validity of calculation.

3) Due to adopting the self localization based on fast Hough transformation, the map built on-line can be revised real-time. Moreover, this approach of self localization overcomes the shortcoming of high computation amount and low precision of localization with classical Hough transformation. Of course this approach also has some limitations including high requirements for environment, a lot of feature straight lines needed, no or less dynamic disturbance and so on.

4) The reconstructed map has higher precision with the new tool. Shown in Fig. 8 we can see that there are still many noises in the map. So we are considering a new tool coupling with other information filters as a pretreatment to reduce the noises and improve the quality of map.

\section{CONCLUSION}

In this paper we present a new tool to reconstruct map for autonomous mobile robot running in a static environment. These outline features including circle, rectangle, and triangle can be reconstructed clearly. This new tool provides an approach for studying autonomous navigation of mobile robot or unmanned land vehicle (ULV) in unknown environment, 
also provides a human-computer interactive interface to manage and manipulate the robot remotely. The mentioned method of self localization overcomes some shortcomings of classical Hough transform. However it is difficult to overcome the natural shortcoming of needing lots of feature straight lines. So we must mix it with other methods in complex environment. Since the PCR6 fusion rule proposed recently by Martin and Osswald [16] has already been shown in another application more efficient than PCR5 and many other classical fusion rules (i.e Dempster's rule[5], Dubois \& Prade's rule[17], etc). We are carrying out a further research on DSmC rule coupled with PCR6 and considering information filters as a pretreatment task in order to reduce the dynamic noises and improve the quality of map.

\section{ACKNOWLEDGMENTS}

This work is supported by National Natural Science Foundation of China (No.60675028). The authors are grateful to Dr. Jean Dezert from ONERA/DTIM, Châtillon, France and Professor Florentin Smarandache from Department of mathematics, University of New Mexico, Gallup, USA, for their helps and technical supports in DSmT and PCR.

\section{REFERENCES}

[1] Elfes, H. Moravec, "High resolution maps from wide angle sonar", IEEE Int Conf Robotics and Automation, pp.116-121, 1985.

[2] Elfes.Using occupancy grids for mobile robot perception and navigation. Computer, 1989, 22(6):46 57

[3] G .Oriolo, G. Ulivi, Vendittelli M. "Fuzzy maps: A new tool for mobile robot perception and planning". Joural of Robotic System 1997,14(3):179 197.

[4] P. Daniel et al. "An evidential approach to probabilistic map-building". in: Proceedings of IEEE Int. Conf. on Robotics and Automation, Minneapolis, Minnesota, April, 1996:745 750

[5] G.. Shafer. "A mathematical theory of evidence. Princeton", N.J. Princeton University Press, 1976

[6] W.Wang et al. "Uncertainty Sensor Information Processing in Map Building of Mobile Robot". ACTA AUTOMATICA SINICA 2003,29(2):270 274.

[7] F. Smarandache, J. Dezert (Editors), "Applications and Advances of DSmT for Information Fusion", Amer. Res. Press, Rehoboth, 2004, http://www.gallup.unm.edu/ - smarandache/DSmTbook1.pdf.

[8] Li, X, Huang. M. Wang "Robot Map Reconstruction from Sonar Sensors and DSmT" Inf. \& Sec.: An Int. J., Bulg. Acad. of Sci., Sofia, 2006,20:104 121.

[9] X. Li, X, Huang. M. Wang. "Sonar Grid Map Reconstruction Of Mobile Robots Based on DSmT". Information Technology Journal 2006. 5(2):267-272.

[10] X. Li, X, Huang et al. "A Comparision of The Effect of Sonar Grid Map Reconstruction Based on DSmT and DST". Proceeding of IEEE WCICA2006. Dalian China. June, 2006,5:4073 4077.

[11] X. Li, X, Huang et al. "A successful application of DSmT in sonar grid map building and comparison with DST-based approach" International Journat of Innovative Computing, Information and Control, 2007, 3(3) in print.

[12] F. Smarandache, J. Dezert, "Information Fusion Based on New Proportional Conflict Redistribution Rules", Proc. of Fusion 2005, July 2005.

[13] R. O. Duda, P. E. Hart. "Use of the HT to detect lines and curves in pictures". Comm. ACM, 1972, 15: 211 215.

[14] H. Lu, Z. Zheng, "New fast Hough transform for line detection "Computer Applications, 2005, 25(10):2379 2380.
[15] F. Fan, D.Ma, Z,Dai. "Mobile Robot Global Localization Based on Model Matching in Hough Space". Robot, 2005, 27(1):35 40

[16] A. Martin, C. Osswald. "A new generalization of the proportional conflict redistribution rule stable in terms of decision", Chapter in Applications and Advances of DSmT for Information Fusion, edited by F. Smarandache, J. Dezert, Amer. Res. Press, Rehoboth, 2006, http://www.gallup.unm.edu/ smarandache/DSmTbook2.pdf.

[17] Dubois D., Prade H., "Representation and com-bination of uncertainty with belief functions and possibility measures," Comp. Intell., 1988, 4:244-264. 